\title{
The importance of different timings of excitatory and inhibitory pathways in neural field models
}

\author{
Carlo R. Laing* \\ Institute of Information and Mathematical Sciences, Massey University, \\ Private Bag 102-904 North Shore Mail Centre, Auckland, New Zealand \\ and \\ S. Coombes ${ }^{\dagger}$ \\ Department of Mathematical Sciences, \\ University of Nottingham, Nottingham, NG7 2RD, UK.
}

December 23, 2005

Running title: "Different timings in neural field models"

Corresponding author:

Carlo R. Laing,

Institute of Information and Mathematical Sciences,

Massey University, Private Bag 102-904 North Shore Mail Centre,

Auckland, New Zealand

ph: +64-9-4140800 extn 41038

fax: +64-9-441-8136

email: c.r.laing@massey.ac.nz

*email: c.r.laing@massey.ac.nz

$\dagger$ email: stephen. coombes@nottingham.ac .uk 


\title{
The importance of different timings of excitatory and inhibitory pathways in neural field models
}

\begin{abstract}
In this paper we consider a neural field model comprised of two distinct populations of neurons, excitatory and inhibitory, for which both the velocities of action potential propagation and the time courses of synaptic processing are different. Using recently-developed techniques we construct the Evans function characterising the stability of both stationary and travelling wave solutions, under the assumption that the firing rate function is the Heaviside step. We find that these differences in timing for the two populations can cause instabilities of these solutions, leading to, for example, stationary breathers. We also analyse "anti-pulses," a novel type of pattern for which all but a small interval of the domain (in moving coordinates) is active. These results extend previous work on neural fields with space-dependent delays, and demonstrate the importance of considering the effects of the different time-courses of excitatory and inhibitory neural activity.
\end{abstract}




\section{The importance of different timings of excitatory and inhibitory pathways in neural field models}

\section{Introduction}

Models of neural fields have been studied extensively over the last few decades $[5,19,32,33$, $13,30,6,7]$, in the hope that they will provide information about possible macroscopic patterns of activity in the cortex. These patterns are on a much larger spatial scale than that of individual neurons, so the models take a continuum limit in which space is continuous and the mean firing rate of neurons is the variable of interest. Among some of the patterns of interest are stationary, spatially-localised "bumps" of activity $[1,33,30]$. These are thought to be involved in working memory and feature selectivity in the visual system [19]. Travelling waves of activity are also of interest. Experimentally, these can be induced by stimulating pharmacologically treated neural tissue slices [20,32]; they have also been observed in several different areas of the cortex of awake animals, often when no stimulation is present [10]. An important question is: Given a neural model, what sorts of spatiotemporal patterns can exist and which features of the model are important in determining the existence and stability of these patterns? In this paper we answer these questions, concentrating on delays and differences in timing relating to neural processing.

It is well-known that different types of synaptic events have quite different time-courses. For example, inhibitory $\mathrm{GABA}_{A}$ synapses decay with a time constant of approximately 15ms, while excitatory NMDA synapses have a time constant of approximately 80ms [27]. Also, the speed of propagation of an action potential along an axon depends on the diameter of the axon, as well as whether it is myelinated or not [27]. Measured axonal conduction velocities in the cortex can differ by a factor of 10, depending on the type of neuron examined, and can be as low as 1m/s [38]. These velocities are also use-dependent and can change over time [37]. Interestingly, anaesthetics have been shown to increase conduction velocity in myelinated fibres - the consequences of this are discussed in [40]. In this paper we study a neural field model that explicitly includes differences in timing (in both the speed of synaptic processing and the conduction speed) for two populations of neurons, excitatory and inhibitory.

Many authors modelling neural systems have assumed instantaneous communication between different parts of the domain $[19,33,13,30]$, but recently the effects of delays due to finite conduction speeds and synaptic processing have been investigated. Some of these more recent studies have involved rate models, in which the firing rate of neurons is the variable of interest $[6,7,32,22,21]$, while several others have investigated networks of spiking neurons $[2,15,17]$. In all of these papers only one type of neuron is considered, and hence there is only one delay. (Golomb and Ermentrout [17] consider both excitatory and 
inhibitory neurons, but only one conduction velocity.) In this paper we study the more realistic case of a network of excitatory and inhibitory neurons, each of which have their own associated conduction velocities (and therefore delays), and their own time-constants for synaptic processing. We would like to know whether this extra level of complexity brings with it any new phenomena that cannot occur in simpler models.

The structure of the paper is as follows. In Sec. 2 we discuss the model integral equations and show that for certain choices of connectivity functions, they can also be formulated as PDEs. In Sec. 3 we discuss stationary (i.e. time-independent) localised solutions, while we discuss travelling solutions in Sec. 4. In both cases we use an Evans function approach [7] to determine the stability of solutions and the conditions necessary for both drift and breathing bifurcations. In Sec. 5 we examine the collision of two fronts, which leads us to study anti-pulses. We also briefly consider networks in which excitatory connections have longer spatial range than inhibitory ones. We finish with a discussion in Sec. 6.

\section{The model}

We analyze a neural field model with synaptic activity $u=u_{e}(x, t)-u_{i}(x, t), x \in \mathbb{R}, t \in \mathbb{R}^{+}$, where $u_{a}, a \in\{e, i\}$, is given by the integral equation

$$
\begin{aligned}
u_{a} & =\eta_{a} * \psi_{a}, \\
\psi_{a}(x, t) & =\int_{-\infty}^{\infty} \mathrm{d} y w_{a}(y) f \circ u\left(x-y, t-|y| / v_{a}\right)
\end{aligned}
$$

where " $\mathrm{e}$ " and " $\mathrm{i}$ " label excitatory and inhibitory populations, respectively. Here, the symbol $*$ represents a temporal convolution in the sense that

$$
(\eta * f)(x, t)=\int_{0}^{t} \eta(s) f(x, t-s) \mathrm{d} s .
$$

The function $\eta_{a}(t)$ (with $\eta_{a}(t)=0$ for $t<0$ ) represents a synaptic filter, whilst $w_{a}(x)=w_{a}(|x|)$ is a synaptic footprint describing the anatomy of network connections and $v_{a}$ is the speed of axonal transmission for population $a$. The function $f$ represents the firing rate of a single

neuron. For the rest of this paper we shall take

$$
w_{a}(x)=\frac{\Gamma_{a}}{2 \sigma_{a}} \mathrm{e}^{-|x| / \sigma_{a}}
$$

often choosing $\Gamma_{e}=1$ and $\Gamma_{i}=\Gamma$. Thus $\Gamma$ is a measure of the strength of the inhibitory population relative to that of the excitatory. It should be noted that Golomb and Ermentrout [15] found some qualitatively different results in systems with exponential coupling (as above) as opposed to systems with other forms of coupling (Gaussian, square), and the same may happen for the system studied here.

The interpretation of this model is that it is the value of the synaptic activity, $u(x, t)$, that determines whether neurons at position $x$ are firing at time $t$. If they are, action potentials 
from population $a$ then propagate at speed $v_{a}$ to other positions in the network, where they are synaptically filtered by other neurons in population $a$ with function $\eta_{a}$. Thus we have assumed that interactions of this form within a population are much stronger than those between populations. The two populations only interact through the difference, $u_{e}-u_{i}$, driving both populations. This model is novel in its consideration of different conduction velocities for the two types of neuron. In Sec. 6 we discuss a more general model, in which we have different firing rate functions for both populations and the possibility of synaptic interactions between the two populations of neurons, not just within them.

This non-local description of neural tissue neglects local delays (say within a hypercolumn of spatial scale $1 \mathrm{~mm}$ ), though incorporates delays due to the finite velocity of action potential propagation between distinct cortical regions. For conduction velocities in the range $1.5-7 \mathrm{~m} / \mathrm{s}$ (typical of white matter axons) these are expected to be significant over scales ranging from a single cortical area (of spatial scale $10 \mathrm{~mm}$ ) up to the scale of interhemispherical collosal connections. Anatomical surveys show that $80 \%$ of the synapses of long-range lateral connections connect directly between pyramidal cells, which are thought to make excitatory synapses only [31]. The other $20 \%$ of the connections target inhibitory interneurons which in turn contact the pyramidal cells, and thus represent inhibitory connections. Even though the inhibitory connections are outnumbered, the net effect of having two distinct delayed pathways has often been ignored in modelling studies.

\subsection{A PDE description}

For the particular choice of synaptic footprint (4) it is possible to obtain an equivalent PDE description of the integral equation (2), using ideas developed by Jirsa and Haken [23]. To see this we write

$$
\psi_{a}(x, t)=\int_{-\infty}^{\infty} \int_{-\infty}^{\infty} G_{a}(x-y, t-s) \rho(y, s) \mathrm{d} s \mathrm{~d} y
$$

where

$$
G_{a}(x, t)=\delta\left(t-|x| / v_{a}\right) w_{a}(x)
$$

and we use the notation $\rho(x, t)=f \circ u(x, t)$. Introducing Fourier transforms of the following form

$$
\psi_{a}(x, t)=\frac{1}{(2 \pi)^{2}} \int_{-\infty}^{\infty} \int_{-\infty}^{\infty} \mathrm{e}^{-i(k x+\omega t)} \psi_{a}(k, \omega) \mathrm{d} k \mathrm{~d} \omega
$$

allows us to write

$$
\psi_{a}(k, \omega)=G_{a}(k, \omega) \rho(k, \omega) .
$$

It is straightforward to show that the Fourier transform of (6) is

$$
G_{a}(k, \omega)=\nu_{a}\left(\omega / v_{a}+k\right)+\nu_{a}\left(\omega / v_{a}-k\right)
$$

where

$$
\nu_{a}(E)=\int_{0}^{\infty} w_{a}(x) \mathrm{e}^{-i E x} \mathrm{~d} x=\left(\frac{\Gamma_{a}}{2 \sigma_{a}}\right) \frac{1}{\sigma_{a}^{-1}+i E}
$$


We have using (9) and (10) that

$$
G_{a}(k, \omega)=\frac{\Gamma_{a}\left(1+i \omega / \omega_{a}\right)}{\left(1+i \omega / \omega_{a}\right)^{2}+\sigma_{a}^{2} k^{2}},
$$

where $\omega_{a}=v_{a} / \sigma_{a}$. We may now write (8) as

$$
\left\{\left(1+i \omega / \omega_{a}\right)^{2}+\sigma_{a}^{2} k^{2}\right\} \psi_{a}(k, \omega)=\Gamma_{a}\left(1+i \omega / \omega_{a}\right) \rho(k, \omega)
$$

which upon inverse Fourier transforming gives the PDE:

$$
\partial_{t t} \psi_{a}+\left(\omega_{a}^{2}-v_{a}^{2} \partial_{x x}\right) \psi_{a}+2 \omega_{a} \partial_{t} \psi_{a}=\Gamma_{a}\left(\omega_{a}^{2}+\omega_{a} \partial_{t}\right) \rho
$$

If we choose the synaptic response $\eta_{a}(t)$ to be the exponential: $\eta_{a}(t)=\alpha_{a} \Theta(t) \exp \left(-\alpha_{a} t\right)$, where $\Theta(t)$ is the Heaviside function, defined by $\Theta(t)=1$ for $t \geq 0$ and zero otherwise, we can also write the integral equation (1) as the differential equation

$$
\frac{1}{\alpha_{a}} \frac{\partial u_{a}}{\partial t}=-u_{a}(x, t)+\psi_{a}(x, t)
$$

In numerical simulations of the model we integrate (13) and (14) using finite difference approximations to the spatial derivatives and work with the choice

$$
f(u)=\frac{1}{1+\mathrm{e}^{-\beta(u-h)}},
$$

where $h$ can be thought of as a threshold and $\beta$ as a gain parameter.

Note that if we set $v_{e}=v_{i}=v$ and $\eta_{e}=\eta_{i}=\eta$, i.e. we remove the differences in timings for the two neural populations, our system reduces to

$$
\begin{aligned}
u & =\eta * \psi \\
\psi(x, t) & =\int_{-\infty}^{\infty} \mathrm{d} y w(y) f \circ u(x-y, t-|y| / v)
\end{aligned}
$$

where $w(y) \equiv w_{e}(y)-w_{i}(y)$, which is typically of "Mexican hat" shape. This equation has been studied elsewhere $[32,6,7]$, and provides a useful comparison. We now consider stationary spatially-localised patterns, or "bumps."

\section{Stationary bump solutions}

For general time-independent solutions, Eqn. (14) gives $u_{a}(x, t)=\psi_{a}(x, t)$, and $u(x, t)$ can be replaced by $q(x)$, where $q(x)$ satisfies

$$
q(x)=\int_{-\infty}^{\infty} \mathrm{d} y w(y) f \circ q(x-y),
$$

where $w(x)=w_{e}(x)-w_{i}(x)$. Throughout the rest of this paper we shall focus on the particular choice of a Heaviside firing rate function, $f(u)=\Theta(u-h)$. In this case, once the position 
of the threshold crossings are known, the explicit dependence on $q$ of the right hand side of (18) is removed. Apart from allowing an explicit construction of travelling bumps and waves this choice also allows for a direct calculation of wave stability via the construction of an Evans function [7]. Although often chosen for mathematical reasons the Heaviside function may be regarded as a natural restriction of sigmoidal functions (such as (15)) to the regime of high gain $(\beta \rightarrow \infty)$. Importantly, numerical simulations show that many of the qualitative properties of solutions in the high gain limit are retained with decreasing gain $[11,32,6]$.

\subsection{Existence}

One-bump solutions with $q(x) \geq h$ for $0<x<\Delta$ are given by

$$
q(x)=\int_{x-\Delta}^{x} \mathrm{~d} y w(y)= \begin{cases}\frac{1}{2}\left\{\left(\mathrm{e}^{x / \sigma_{e}}-\mathrm{e}^{(x-\Delta) / \sigma_{e}}\right)-\Gamma\left(\mathrm{e}^{x / \sigma_{i}}-\mathrm{e}^{(x-\Delta) / \sigma_{i}}\right)\right\} & x<0 \\ \frac{1}{2}\left\{\Gamma\left(\mathrm{e}^{(x-\Delta) / \sigma_{i}}+\mathrm{e}^{-x / \sigma_{i}}-2\right)-\left(\mathrm{e}^{(x-\Delta) / \sigma_{e}}+\mathrm{e}^{-x / \sigma_{e}}-2\right)\right\} & 0 \leq x \leq \Delta . \\ \frac{1}{2}\left\{\left(\mathrm{e}^{-(x-\Delta) / \sigma_{e}}-\mathrm{e}^{-x / \sigma_{e}}\right)-\Gamma\left(\mathrm{e}^{-(x-\Delta) / \sigma_{i}}-\mathrm{e}^{-x / \sigma_{i}}\right)\right\} & x>\Delta\end{cases}
$$

Note that the system (18) is translationally invariant, so we can choose one end point of the bump to be at $x=0$. The width of the bump is then determined by the self-consistent solution of $q(0)=h=q(\Delta)$, which gives the equation:

$$
h=\frac{1-\mathrm{e}^{-\Delta / \sigma_{e}}}{2}-\frac{\Gamma\left(1-\mathrm{e}^{-\Delta / \sigma_{i}}\right)}{2} .
$$

In Fig. 1 we show a typical plot of bump width $\Delta$ as a function of the threshold parameter $h$, for particular values of the other parameters. We note that the differences in timings for the two populations do not affect the existence of stationary patterns, only their stability, as seen below.

\subsection{Stability via the Evans function}

We can find the stability of stationary one-bump solutions by constructing the associated Evans function. Evans functions were first developed to study the stability of travelling waves in PDEs [12]. In essence the Evans function is an analytic tool whose zeros correspond to eigenvalues of the linearised problem obtained after considering perturbations around a travelling wave solution. Moreover, the order of the zero and the multiplicity of the eigenvalue match. A recent review of their use in determining the stability of travelling pulses in dissipative systems can be found in [25]. This theory has recently been extended to cover travelling waves in systems with nonlocal interactions, such as studied here $[26,7,34]$. 


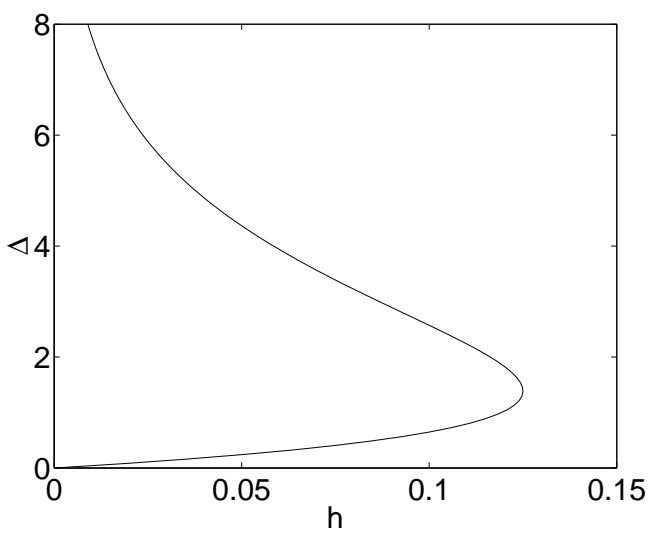

Figure 1: A plot showing stationary bump width $\Delta$ as a function of the threshold parameter $h$, as determined by solving (20). Parameters are $\sigma_{e}=1 \sigma_{i}=2$, and $\Gamma=1$. 
Generalising results in [7] we may construct the Evans function of the stationary onebump solution as $\mathcal{E}(\lambda)=\operatorname{det}(\mathcal{A}(\lambda)-I)$, where

$$
\mathcal{A}(\lambda)=\left[\begin{array}{ll}
A(0, \lambda) & A(\Delta, \lambda) \\
A(\Delta, \lambda) & A(0, \lambda)
\end{array}\right]
$$

where $A(\xi, \lambda)=A_{e}(\xi, \lambda)-A_{i}(\xi, \lambda)$ and

$$
A_{a}(\xi, \lambda)=\frac{1}{\left|q^{\prime}(0)\right|} \widehat{\eta}_{a}(-i \lambda) w_{a}(\xi) \mathrm{e}^{-\lambda \xi / v_{a}}, \quad \widehat{\eta}_{a}(k)=\int_{-\infty}^{\infty} \eta_{a}(x) \mathrm{e}^{-i k x} \mathrm{~d} x .
$$

For our choice of an exponential synaptic response $\eta_{a}(t)=\alpha_{a} \Theta(t) \exp \left(-\alpha_{a} t\right)$, we have simply that

$$
\widehat{\eta}_{a}(-i \lambda)=\frac{1}{1+\lambda / \alpha_{a}}
$$

Note that we can obtain $q^{\prime}(0)$ by using

$$
q^{\prime}(x)=w_{e}(x)-w_{e}(x-\Delta)-\Gamma\left[w_{i}(x)-w_{i}(x-\Delta)\right]
$$

The discrete spectrum for the one-bump solution is then given by the zeros of the Evans function $\mathcal{E}(\lambda)=0$, so that a solution is stable if the spectrum only resides in the left hand complex plane (i.e. $\operatorname{Re} \lambda<0$ ). Note that a zero eigenvalue (satisfying $\mathcal{E}(0)=0$ ) is always expected due to translational invariance of the one-bump solution (with the corresponding eigenfunction $q^{\prime}(x)$ ). One natural way to find the zeros of $\mathcal{E}(\lambda)$ is to write $\lambda=\nu+i \omega$ and plot the zero contours of $\operatorname{Re} \mathcal{E}(\lambda)$ and $\operatorname{Im} \mathcal{E}(\lambda)$ in the $(\nu, \omega)$ plane. The Evans function is zero where the contours intersect. Note that it is sufficient for us to determine the location of the isolated spectrum for wave stability, since the systems under study in this paper are such that the real part of the continuous spectrum has a uniformly negative upper bound [7].

\subsection{Examples}

We now show several examples. If $\sigma_{e}=1, \sigma_{i}=2$ and $\Gamma=1$, we see from Fig. 1 that at $h=0.1$ we have two stationary bumps with widths $\Delta=0.64701$ and $\Delta=2.5719$. If we set $\alpha_{e}=\alpha_{i}=1$ and $v_{i}=1$ and decrease $v_{e}$, we find that the wide stationary bump becomes unstable due to a single eigenvalue passing from the left half plane to the right half plane along the real axis. This is shown in Fig. 2, where we plot contours of the real and imaginary parts of the Evans function over part of the complex $(\lambda)$ plane for $v_{e}=0.25$ and $v_{e}=0.15$. The Evans function has zeros where the two contours cross. Note that we always have a zero at the origin, as expected. This instability manifests itself as a transition to a moving, or drifting, bump, as shown in Fig. 3. Here we run the system with $v_{e}=0.25$ up until $t=100$, when we switch to $v_{e}=0.15$, and add a small random perturbation to the solution. We see that the previously stable stationary bump is replaced by a stable moving bump. If the position of a bump of activity is used to encode some aspect of an action to be performed in the future [14], this 

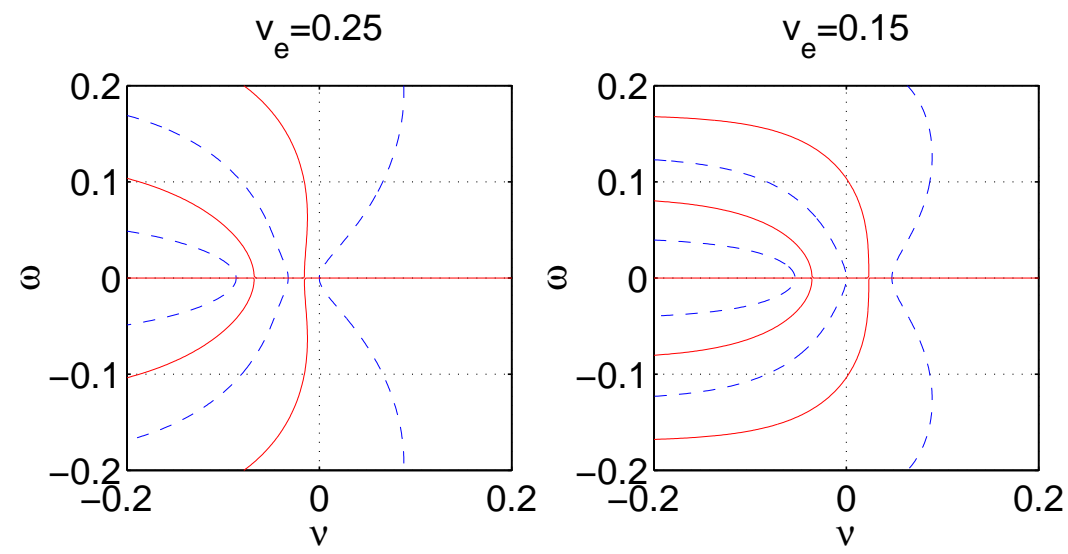

Figure 2: Zero contours of the real part (blue, dashed) and the imaginary part (red, solid) of the Evans function of a stationary bump for $v_{e}=0.25$ (left) and $v_{e}=0.15$ (right). Other parameters are $\sigma_{e}=1, \sigma_{i}=2, \Gamma=1, \alpha_{i}=\alpha_{e}=1, h=0.1, v_{i}=1$. 


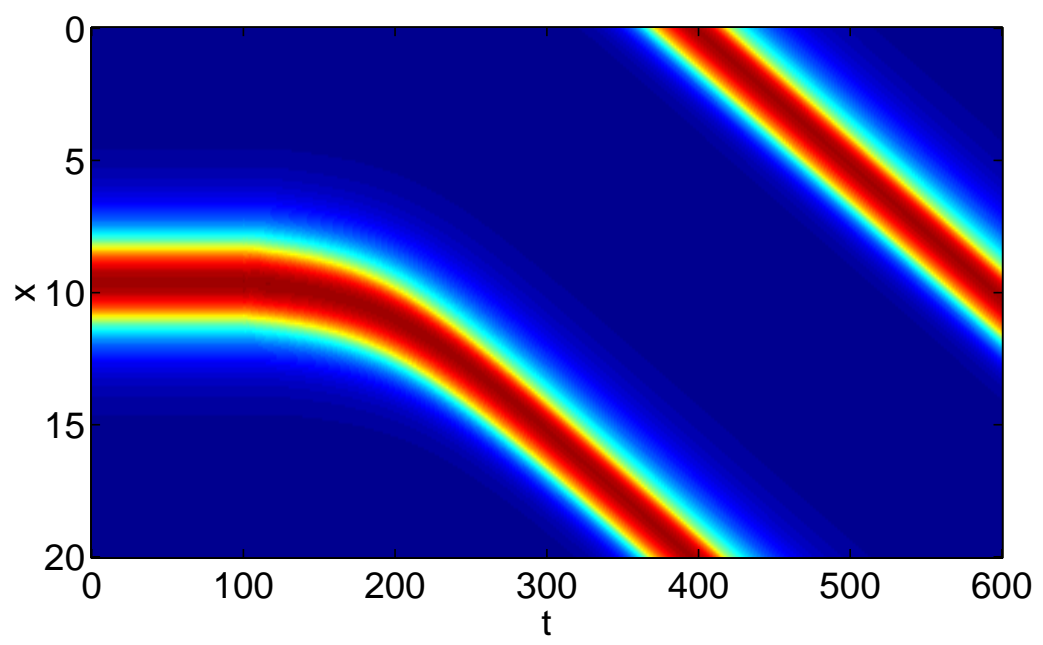

Figure 3: Instability of a stationary bump due to an eigenvalue passing through zero. At $t=100, v_{e}$ was changed from 0.25 to 0.15 (and a small random perturbation was added). $u_{e}$ is plotted, red is high, blue is low. Other parameters are $\sigma_{e}=1, \sigma_{i}=2, \Gamma=1, \alpha_{i}=\alpha_{e}=$ $1, h=0.1, v_{i}=1$. The domain was discretised with 200 spatial points and periodic boundary conditions were used. 
instability is undesirable. For simulations of the full system we use (15) as the firing rate function with $\beta=150$. This moving bump will be discussed later in Sec. 4.2.

Conversely, by holding $v_{e}$ constant and decreasing $v_{i}$ we can make the wide stationary bump in Fig. 1 go unstable through a Hopf bifurcation, in which a pair of complex eigenvalues pass through the imaginary axis. This is shown in Fig. 4, where the Evans function is represented for $v_{i}=0.4$ (left) and $v_{i}=0.2$ (right). We see that between these values, a complex pair of eigenvalues moves into the right half plane. The instability is shown in Fig. 5, where we switch $v_{i}$ from 1 to 0.2 halfway through the simulation. We see an oscillatory instability develop, but it appears to be subcritical, and the system moves to the all-off state. Clearly this is also undesirable in the context of working memory.

These instabilities are summarised in Fig. 6, where we plot the curves of drift instabilities and Hopf bifurcations in the $v_{e}-v_{i}$ plane. Note that we have followed only the curve of Hopf bifurcations corresponding to the imaginary roots with smallest imaginary part. Although we have not proven it, numerical computation of the Evans function at various points in the $v_{e}-v_{i}$ plane suggests that the stationary bump is always stable in the wedge between the two curves.

We can also find supercritical Hopf bifurcations if we relax the restriction that $\alpha_{i}=\alpha_{e}=$ 1 , i.e. if we allow different time-courses for the synaptic processing in the two populations. In Fig. 7 we show the effect of decreasing $v_{e}$ from 0.8 to 0.5 , where $\alpha_{i}=1.8$ and $\alpha_{e}=3$. Oscillations appear, and their amplitude rapidly saturates. Presumably, by changing other parameters it would be possible to observe higher codimension bifurcations, such as the Takens-Bogdanov (double zero eigenvalue) [9].

\subsection{Discussion}

It is possible to show that bifurcations of a stationary bump with an eigenvalue passing through zero are not possible for (1)-(2) if $v_{e}=v_{i}, \alpha_{e}=\alpha_{i}$, and $\sigma_{e}<\sigma_{i}$ (as here) i.e. this is a novel instability due to the differences in timings for the two populations. To show this, one calculates $\mathcal{E}(\lambda)$ and obtains

$$
\mathcal{E}(\lambda)=\frac{1}{(1+\lambda / \alpha)^{2}} \frac{1}{\left|q^{\prime}(0)\right|^{2}}\left\{[(1+\lambda / \alpha) w(\Delta)-(\lambda / \alpha) w(0)]^{2}-\left[w(\Delta) e^{-\lambda \Delta / v}\right]^{2}\right\}
$$

where $v_{e}=v_{i}=v, \alpha_{e}=\alpha_{i}=\alpha$, and we have used the fact that $w(0)>w(\Delta)$. Clearly $\mathcal{E}(0)=0$. If $w(\Delta)<0$, we also have that $\mathcal{E}^{\prime}(\lambda)>0$ for all $\lambda>0$, and hence there can be no other positive real roots of $\mathcal{E}(\lambda)$. The condition $w(\Delta)<0$ means that we are on the upper branch in Fig. 1.

Similarly, it is possible to show that there are no purely imaginary roots of $\mathcal{E}(\lambda)$. We substitute $\lambda=i \omega$ into (25), where $\omega \in \mathbb{R}, \omega \neq 0$ and set the real and imaginary parts equal to 

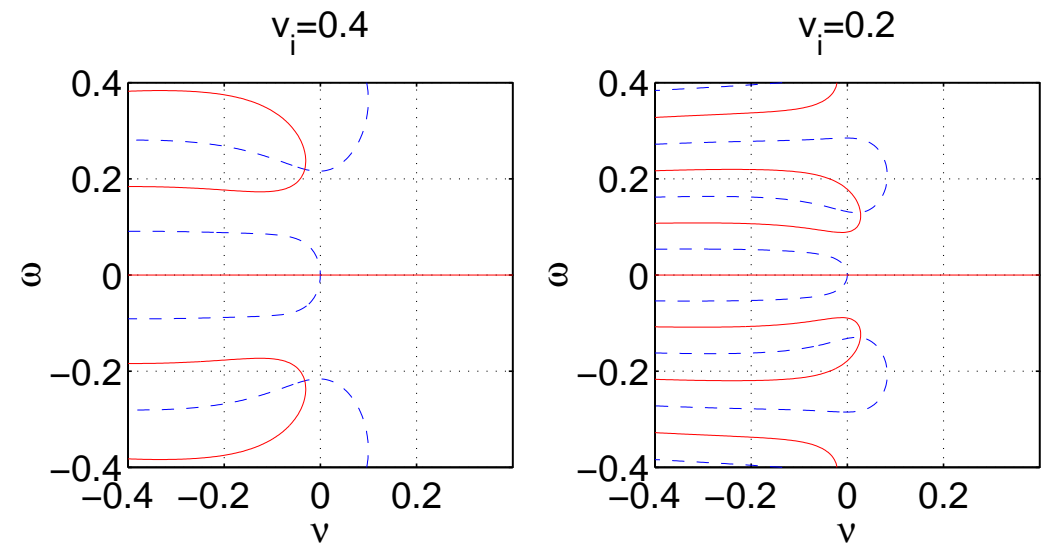

Figure 4: Zero contours of the real part (blue, dashed) and the imaginary part (red, solid) of the Evans function for a stationary bump for $v_{i}=0.4$ (left) and $v_{i}=0.2$ (right). Other parameters are $v_{e}=1, \alpha_{i}=\alpha_{e}=1, h=0.1, \sigma_{e}=1, \sigma_{i}=2$ and $\Gamma=1$. 


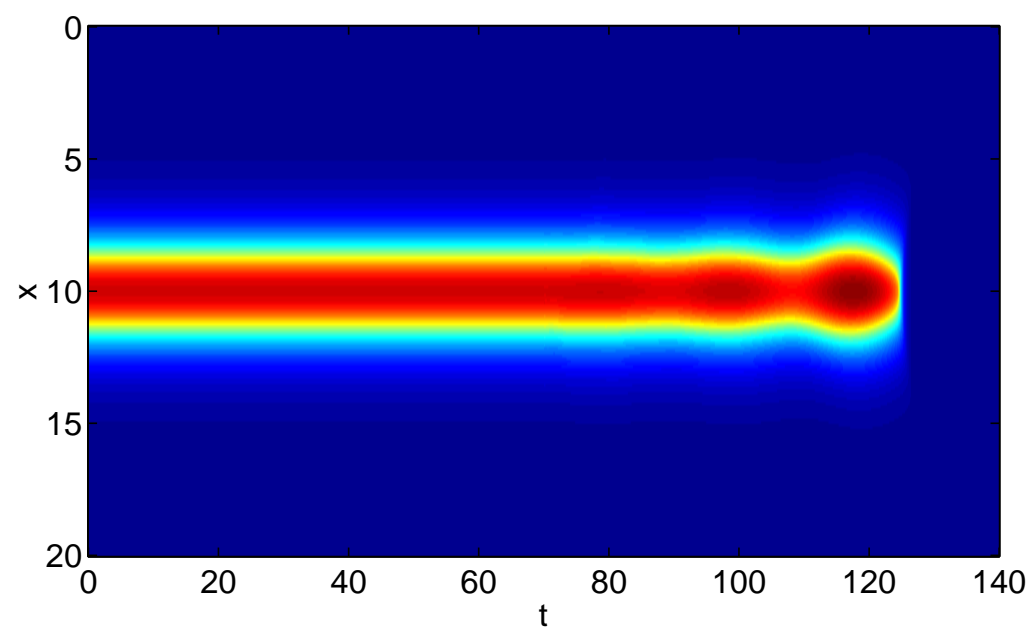

Figure 5: Instability of a stationary bump due to a subcritical Hopf bifurcation. At $t=70$ we switched $v_{i}$ from 1 to 0.2 (and a small random perturbation was added). Other parameters are $v_{e}=1, \alpha_{i}=\alpha_{e}=1, h=0.1, \sigma_{e}=1, \sigma_{i}=2$ and $\Gamma=1 . u_{e}$ is plotted, with red high and blue low. 200 spatial points were used. 


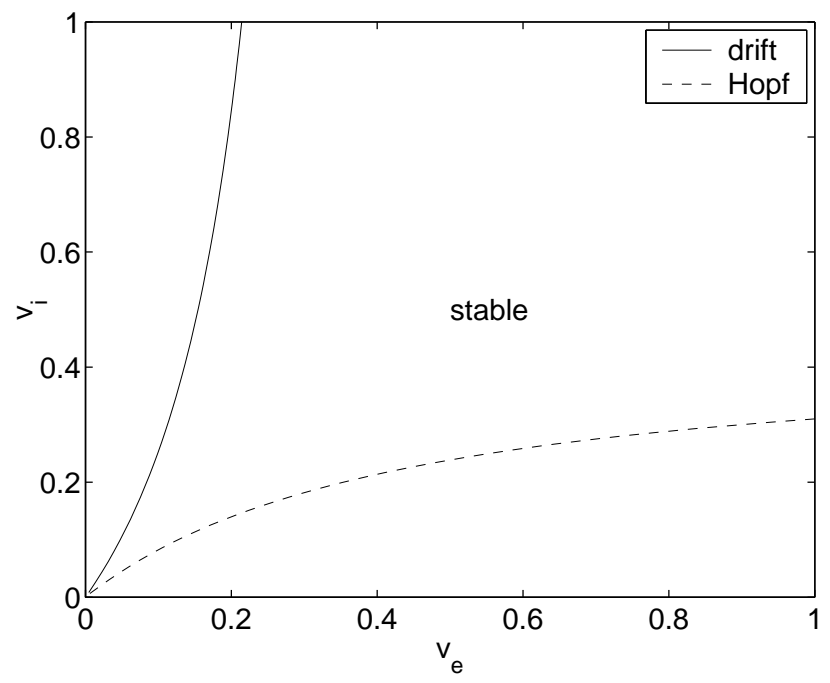

Figure 6: The curve of drift instabilities, defined by $\mathcal{E}^{\prime}(0)=0$ (solid), and the curve of Hopf bifurcations, for which $\mathcal{E}(\lambda)$ has a conjugate pair of purely imaginary roots (dashed). Other parameters are $\alpha_{e}=\alpha_{i}=1, h=0.1, \sigma_{e}=1, \sigma_{i}=2$ and $\Gamma=1$. The stationary bump appears to be stable in the wedge between the two curves and unstable otherwise. 


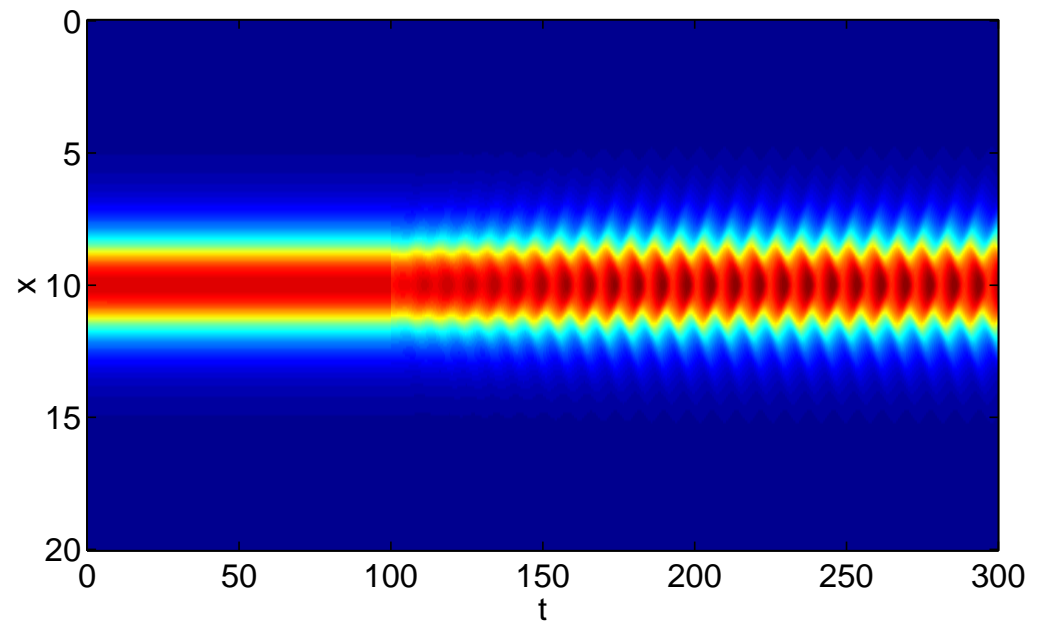

Figure 7: Supercritical Hopf bifurcation of a stationary bump. At $t=100$ we switched $v_{e}$ from 0.8 to 0.5 (and a small perturbation was added). 200 spatial points were used. Other parameters are $\sigma_{e}=1, \sigma_{i}=2, \alpha_{i}=1.8, \alpha_{e}=3, v_{i}=1, h=0.1, \Gamma=1 . u_{e}$ is plotted; red high, blue low. 
zero, obtaining

$$
\begin{array}{r}
{[w(\Delta)]^{2}(1-\cos \theta)-(\omega / \alpha)^{2}[w(\Delta)-w(0)]^{2}=0} \\
w(\Delta)\{w(\Delta) \sin \theta-2(\omega / \alpha)[w(\Delta)-w(0)]\}=0
\end{array}
$$

where $\theta=2 \omega \Delta / v$. Assuming that $w(\Delta) \neq 0$, solving (27) for $w(\Delta)-w(0)$ and substituting into (26) we obtain

$$
[w(\Delta)]^{2}\left[1-\cos \theta-(1 / 4) \sin ^{2} \theta\right]=0
$$

which is only true if $\theta=0,2 \pi, 4 \pi \ldots$ Substituting these values of $\theta$ back into (27) we see that it cannot simultaneously be satisfied, i.e. $\mathcal{E}(\lambda)$ has no purely imaginary roots. If $w(\Delta)=0$ the only root of $\mathcal{E}(\lambda)$ is $\lambda=0$, which corresponds to the saddle-node bifurcation in Fig. 1 . Thus both types of instabilities are a result of the differences in timings for the two populations.

Bifurcations of the types discussed in this section have recently been observed in a system with one neural population but in which the threshold is a dynamic variable [8]. There, the authors also saw a stationary bump start to move as an eigenvalue moved through zero, and stationary breathers caused by a supercritical Hopf bifurcation. Breathers have also been observed in neural field equations by Bressloff et al. [4, 13], but in those papers the authors made the domain inhomogeneous, inducing a bump to occur over a spatially-localised input. During normal awake operation the cortex continuously receives inhomogeneous inputs, so the response of a neural model with such inputs is of interest. However, under general anaesthesia the cortex will receive less input (and conduction velocities may be increased [40]) so it is also of interest to study the existence and stability of patterns in this situation.

Blomquist et al. [1] recently studied a two-layer neural field model that can be best thought of as a generalisation of that studied by Pinto and Ermentrout [33]. In contrast with Pinto and Ermentrout, Blomquist et al. included inhibitory-to-inhibitory connections and did not assume that the firing rate function for the inhibitory population was linear. They did not include conduction delays, but obtained both stable and unstable breathers that were created in Hopf bifurcations, as seen here.

The common theme between our results and those of Coombes and Owen [8], Bressloff et al. [4,13] and Blomquist et al. [1] is the presence of a second variable describing either another population of neurons or a local field such as an adaptation current.

\section{Travelling wave solutions}

We now discuss travelling wave solutions, both fronts (which connect a region of high activity to one of low activity) and pulses (travelling bumps, before and after which the medium is quiescent). We introduce the coordinate $\xi=x-c t$ and seek functions $U(\xi, t)=u(x-c t, t)$ 
that satisfy the full integral model equations. In the $(\xi, t)$ coordinates, these integral equations can be expressed as $U(\xi, t)=U_{e}(\xi, t)-U_{i}(\xi, t)$, with

$$
U_{a}(\xi, t)=\int_{-\infty}^{\infty} \mathrm{d} y w_{a}(y) \int_{0}^{\infty} \mathrm{d} s \eta_{a}(s) f \circ U\left(\xi-y+c s+c|y| / v_{a}, t-s-|y| / v_{a}\right) .
$$

The travelling wave is a stationary solution $U(\xi, t)=q(\xi)$ (independent of $t$ ), that satisfies $q(\xi)=q_{e}(\xi)-q_{i}(\xi)$, with

$$
q_{a}(\xi)=\int_{0}^{\infty} \mathrm{d} s \eta_{a}(s) \psi_{a}(\xi+c s)
$$

where

$$
\psi_{a}(\xi)=\int_{-\infty}^{\infty} \mathrm{d} y w_{a}(y) f \circ q\left(\xi-y+c|y| / v_{a}\right)
$$

\subsection{Fronts}

We look for travelling front solutions such that $q(\xi)>h$ for $\xi<0$ and $q(\xi)<h$ for $\xi>0$. It is then a simple matter to show that (for the special case of the Heaviside firing rate function chosen earlier)

$$
\psi_{a}(\xi)=\left\{\begin{array}{ll}
\int_{\xi /\left(1-c / v_{a}\right)}^{\infty} \mathrm{d} y w_{a}(y)=\Gamma_{a} \exp \left(m_{a}^{-} \xi\right) / 2 & \xi \geq 0 \\
\int_{\xi /\left(1+c / v_{a}\right)}^{\infty} \mathrm{d} y w_{a}(y)=\Gamma_{a}\left[1-\exp \left(m_{a}^{+} \xi\right) / 2\right] & \xi<0
\end{array}, \quad m_{a}^{ \pm}=\frac{\omega_{a}}{c \pm v_{a}}\right.
$$

The choice of origin, $q(0)=h$, gives an implicit equation for the speed of the wave as a function of system parameters:

$$
\begin{array}{rlrl}
2 h & =\frac{1}{1-c m_{e}^{-} / \alpha_{e}}-\frac{\Gamma}{1-c m_{i}^{-} / \alpha_{i}} & & c \geq 0 \\
2 h & =2(1-\Gamma)+\frac{\Gamma}{1-c m_{i}^{+} / \alpha_{i}}-\frac{1}{1-c m_{e}^{+} / \alpha_{e}} & c<0
\end{array}
$$

Both equations may be written as quadratic equations in $c$. To ensure that $\lim _{\xi \rightarrow-\infty} q(\xi)>h$, we must have $\int_{\mathbb{R}} w(y) \mathrm{d} y=1-\Gamma>h$. We note that a standing front $(c=0)$ occurs when $2 h=1-\Gamma$. Also, it is not necessary to have different propagation velocities and synaptic processing time-constants for such fronts to exist; they are seen robustly in simpler systems where these parameters are equal [32].

Once again the Evans function is easily obtained using the techniques described in [7] and may be written in the form

$$
\mathcal{E}(\lambda)=1-\frac{\mathcal{H}(\lambda)}{\mathcal{H}(0)}
$$

where $\mathcal{H}(\lambda)=\mathcal{H}_{e}(\lambda)-\mathcal{H}_{i}(\lambda)$ and

$$
\mathcal{H}_{a}(\lambda)=\int_{0}^{\infty} \mathrm{d} y w_{a}(y) \eta_{a}\left(y / c-y / v_{a}\right) \mathrm{e}^{-\lambda y / c} .
$$

For the exponential synaptic response we have simply that

$$
\mathcal{H}_{a}(\lambda)=\frac{\alpha_{a} \Gamma_{a}}{2 \sigma_{a}}\left[\frac{1}{\sigma_{a}}+\alpha_{a}\left(\frac{1}{c}-\frac{1}{v_{a}}\right)+\frac{\lambda}{c}\right]^{-1} .
$$


For a standing front it is a simple matter to check that $\mathcal{E}(\lambda)=0$ when

$$
\lambda=\frac{\Gamma \alpha_{e} \sigma_{e}-\alpha_{i} \sigma_{i}}{\sigma_{i}-\Gamma \sigma_{e}}
$$

Hence, there is a bifurcation of the standing front when $\Gamma \alpha_{e} \sigma_{e}=\alpha_{i} \sigma_{i}$ and $2 h=1-\Gamma$. To determine the type of bifurcation, one examines various partial derivatives of the functions in (33)-(34), evaluated at the bifurcation point (see, e.g. Sec. 3.1 of [41]). It can be determined that the bifurcation is a simultaneous pair of transcritical bifurcations, each creating a branch with $c \neq 0$. (This bifurcation was misidentified as a pitchfork bifurcation in Refs. [3, 7].) The transcritical bifurcations are shown in Fig. 8 (left) for specific values of the other parameters. When the condition $2 h=1-\Gamma$ does not hold, the transcritical bifurcations generically break into a single saddle-node bifurcation, as seen in Fig. 8 (right). Similar results have been seen before in a one-layer model with a linear recovery variable [7,3]. Although we have not seen Hopf bifurcations of fronts, it is known that making the domain inhomogeneous can induce these [4].

Figure 9 shows another plot of front speed, now as a function of $h$. We see that for these parameter values there is a minimum speed below which stable fronts do not exist. We will return to this figure later in Sec. 5. Finally, we see in Fig. 10 a result of choosing different parameters. For these parameters, only fronts with slow enough speeds are stable. This is in strong contrast with results from networks with purely excitatory coupling $[2,15]$ in which travelling structures cannot travel with arbitrarily slow speeds.

\subsection{Travelling Pulses}

We now study travelling pulses, which are bumps of the form studied in Sec. 3, but which have speed $c \neq 0$. The travelling pulses have the form $q(\xi) \geq h$ for $\xi \in[0, \Delta]$ and $q(\xi)<h$ otherwise. In this case the expression for $\psi_{a}(\xi)$ is given by [6]

$$
\psi_{a}(\xi)= \begin{cases}\mathcal{F}_{a}\left(\frac{-\xi}{1+c / v_{a}}, \frac{\Delta-\xi}{1+c / v_{a}}\right) & \xi \leq 0 \\ \mathcal{F}_{a}\left(0, \frac{\xi}{1-c / v_{a}}\right)+\mathcal{F}_{a}\left(0, \frac{\Delta-\xi}{1+c / v_{a}}\right) & 0<\xi<\Delta, \\ \mathcal{F}_{a}\left(\frac{\xi-\Delta}{1-c / v_{a}}, \frac{\xi}{1-c / v_{a}}\right) & \xi \geq \Delta\end{cases}
$$

where

$$
\mathcal{F}_{a}\left(x_{1}, x_{2}\right)=\int_{x_{1}}^{x_{2}} w_{a}(y) \mathrm{d} y=\frac{\Gamma_{a}\left[\exp \left(-x_{1} / \sigma_{a}\right)-\exp \left(-x_{2} / \sigma_{a}\right)\right]}{2}, \quad x_{2}>x_{1}>0 .
$$

The dispersion relation $c=c(\Delta)$ is then implicitly defined by the simultaneous solution of $q(0)=h$ and $q(\Delta)=h(\Delta>0)$. For an exponential synapse these two conditions are 

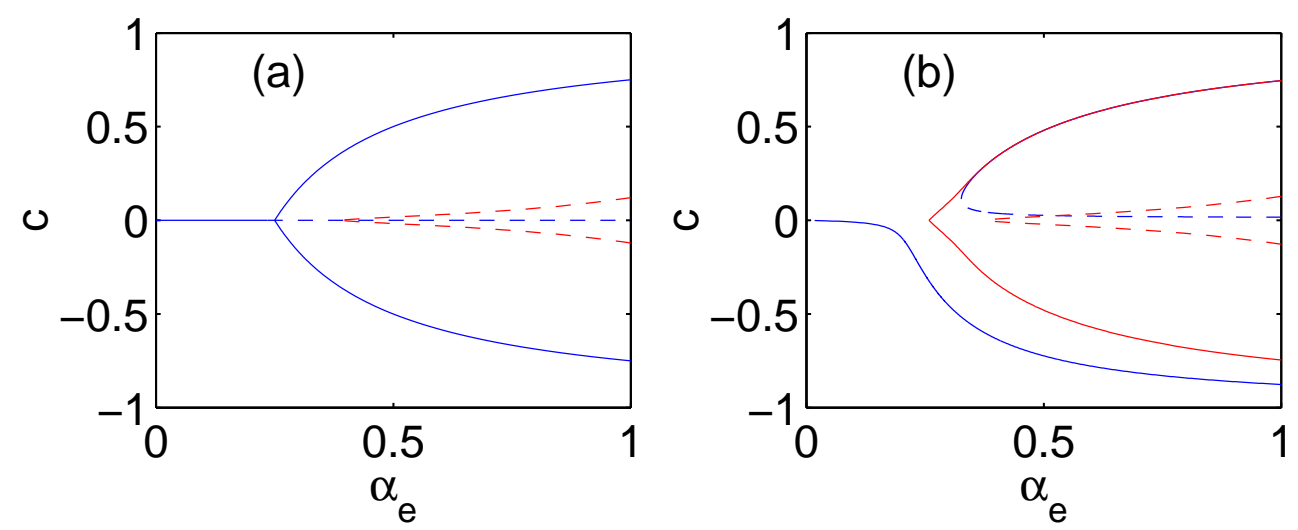

Figure 8: (a): Speed of a front for $2 h=1-\Gamma$, in blue $(h=0.1, \Gamma=0.8)$. Solid lines are stable while dashed are unstable, as determined by the Evans function. Note the pair of transcritical bifurcations at $\alpha_{e}=\alpha_{i} \sigma_{i} /\left(\Gamma \sigma_{e}\right)$. (b): Speed of a front when $2 h \neq 1-\Gamma$, in blue ( $h=0.1, \Gamma=0.85)$. The red curves correspond to travelling pulses, discussed later. For $\Gamma<0.8$, the blue curves in the left panel breal in the opposite sense to those shown in the 


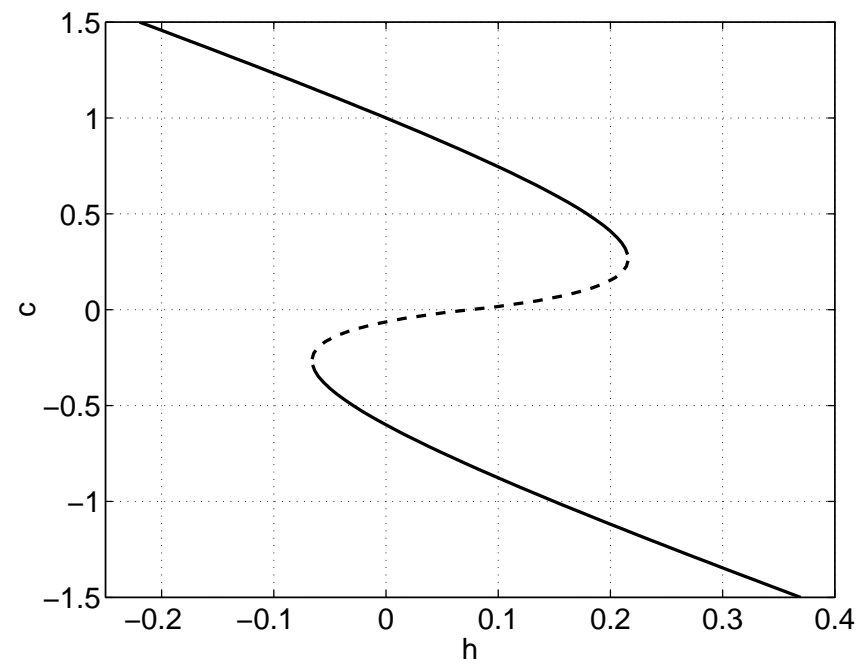

Figure 9: Front speed as a function of $h$. Stable fronts are represented by solid lines, unstable by dashed. Parameters are $\Gamma=0.85, \alpha_{e}=1, \alpha_{i}=0.1, v_{e}=v_{i}=1, \sigma_{i}=2$ and $\sigma_{e}=1$. 


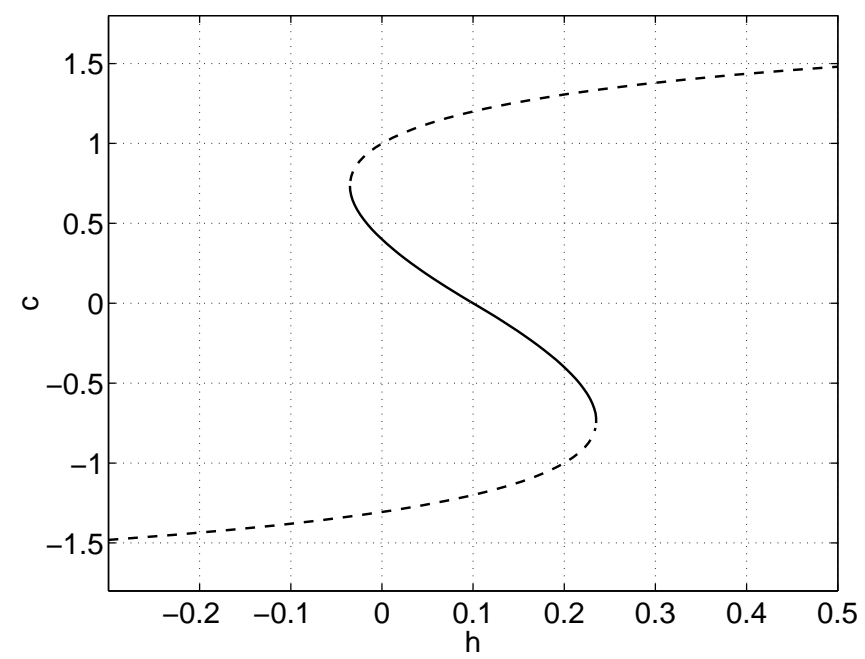

Figure 10: Front speed as a function of $h$. Parameters are $\alpha_{i}=\alpha_{e}=1, v_{e}=v_{i}=1, \sigma_{e}=1, \sigma_{i}=$ $2, \Gamma=0.8$. The solid branch is stable, while the dashed ones are unstable. 


$$
\begin{aligned}
2 h & =2(1-\Gamma)+2\left[\Gamma \exp \left(-\alpha_{i} \Delta / c\right)-\exp \left(-\alpha_{e} \Delta / c\right)\right] \\
& +\left[\frac{\exp \left(-\alpha_{e} \Delta / c\right)-1}{1-c m_{e}^{-} / \alpha_{e}}\right]+\left[\frac{\exp \left(-\alpha_{e} \Delta / c\right)-\exp \left(-m_{e}^{+} \Delta\right)}{1-c m_{e}^{+} / \alpha_{e}}\right] \\
& -\Gamma\left[\frac{\exp \left(-\alpha_{i} \Delta / c\right)-1}{1-c m_{i}^{-} / \alpha_{i}}\right]-\Gamma\left[\frac{\exp \left(-\alpha_{i} \Delta / c\right)-\exp \left(-m_{i}^{+} \Delta\right)}{1-c m_{i}^{+} / \alpha_{i}}\right] \\
2 h & =\left[\frac{1-\exp \left(m_{e}^{-} \Delta\right)}{1-c m_{e}^{-} / \alpha_{e}}\right]-\Gamma\left[\frac{1-\exp \left(m_{i}^{-} \Delta\right)}{1-c m_{i}^{-} / \alpha_{i}}\right]
\end{aligned}
$$

Note that as $c \rightarrow 0$, both (41) and (42) reduce to the equation governing the width of a stationary bump, (20), as expected.

The Evans function takes the same form as in Sec. 3.2, with (21) replaced by

$$
\mathcal{A}(\lambda)=\left[\begin{array}{ll}
A(0, \lambda) & B(0, \lambda) \\
A(\Delta, \lambda) & B(\Delta, \lambda)
\end{array}\right] .
$$

where $A(\xi, \lambda)=A_{e}(\xi, \lambda)-A_{i}(\xi, \lambda), B(\xi, \lambda)=B_{e}(\xi, \lambda)-B_{i}(\xi, \lambda)$, and

$$
\begin{aligned}
& A_{a}(\xi, \lambda)=\frac{1}{c\left|q^{\prime}(0)\right|} \int_{\xi /\left(1-c / v_{a}\right)}^{\infty} \mathrm{d} y w_{a}(y) \eta_{a}\left(-\xi / c+y / c-y / v_{a}\right) \mathrm{e}^{-\lambda(y-\xi) / c} \\
& B_{a}(\xi, \lambda)=\frac{1}{c\left|q^{\prime}(\Delta)\right|} \int_{(\xi-\Delta) /\left(1+c / v_{a}\right)}^{\infty} \mathrm{d} y w_{a}(y) \eta_{a}\left((\Delta-\xi) / c+y / c-|y| / v_{a}\right) \mathrm{e}^{-\lambda(y-(\xi-\Delta)) / c}
\end{aligned}
$$

Explicitly, we have

$$
\begin{aligned}
A_{a}(0, \lambda) & =\frac{\alpha_{a} \Gamma_{a}}{2\left|q^{\prime}(0)\right|\left(c-\alpha_{a} / m_{a}^{-}+\lambda \sigma_{a}\right)} \\
A_{a}(\Delta, \lambda) & =\exp \left[-\Delta\left(v_{a}+\sigma_{a} \lambda\right) /\left(\sigma_{a}\left(v_{a}-c\right)\right)\right] A_{a}(0, \lambda), \\
B_{a}(0, \lambda) & =\frac{\alpha_{a} \Gamma_{a}}{2\left|q^{\prime}(\Delta)\right|}\left\{\frac{\exp \left[-\Delta\left(\alpha_{a}+\lambda\right) / c\right]-\exp \left[-\Delta\left(v_{a}+\lambda \sigma_{a}\right) /\left(\sigma_{a}\left(v_{a}+c\right)\right)\right]}{c-\alpha_{a} / m_{a}^{+}-\lambda \sigma_{a}}\right. \\
& \left.+\frac{\exp \left[-\Delta\left(\alpha_{a}+\lambda\right) / c\right]}{c-\alpha_{a} / m_{a}^{-}+\lambda \sigma_{a}}\right\} \\
B(\Delta, \lambda) & =\left|\frac{q^{\prime}(0)}{q^{\prime}(\Delta)}\right| A(0, \lambda),
\end{aligned}
$$

Note that as $c \rightarrow 0$, the matrix (43) reduces to the matrix associated with the stability of a stationary bump, (21), as expected. To evaluate the derivatives of $q$ in (46)-(49) we use $q^{\prime}=q_{e}^{\prime}-q_{i}^{\prime}$, where $q_{a}^{\prime}=\alpha_{a}\left(q_{a}-\psi_{a}\right) / c$. Specifically, we have

$$
\begin{gathered}
\psi_{a}(0)=\Gamma_{a}\left[1-\exp \left(-m_{a}^{+} \Delta\right)\right] / 2, \quad \psi_{a}(\Delta)=\Gamma_{a}\left[1-\exp \left(m_{a}^{-} \Delta\right)\right] / 2, \\
q_{a}(0)=\Gamma_{a}\left[1-\exp \left(-\alpha_{a} \Delta / c\right)+\frac{1-\exp \left(-\alpha_{a} \Delta / c\right)}{2\left(c m_{a}^{-} / \alpha_{a}-1\right)}+\frac{\exp \left(-m_{a}^{+} \Delta\right)-\exp \left(-\alpha_{a} \Delta / c\right)}{2\left(c m_{a}^{+} / \alpha_{a}-1\right)}\right]
\end{gathered}
$$

and

$$
q_{a}(\Delta)=\frac{\Gamma_{a}\left[1-\exp \left(m_{a}^{-} \Delta\right)\right]}{2\left(1-c m_{a}^{-} / \alpha_{a}\right)}
$$


We now discuss several examples. In Fig. 11 we plot the speeds and widths of a pair of travelling pulses (one stable and one unstable) as $v_{e}$ is varied. The other parameter values are the same as those in Fig. 3, and the instability seen in that figure can can now be understood with reference to Fig. 11. For $v_{e}=0.25$ there is no stable travelling pulse, but for $v_{e}=0.15$ there is one, with speed $c \approx 0.05$. (Of course, a similar stable pulse moving in the opposite direction also exists.) Interestingly, this pair of stable pulses seem to be created in a pair of transcritical bifurcations, in the same way that fronts were created in Sec. 4.1. This is in contrast with the pitchfork bifurcation in speed seen in other neural field models [29]. Note that for these parameters a moving front does not exist, as the condition $1-\Gamma>h$ does not hold.

In Fig. 12 we show the width and speed of a moving pulse as a function of $h$. For a range of values of $h$, there are two pulses, a fast, wide one and a slow, narrow one. By plotting the Evans function on the upper branch (not shown) we see that there is a Hopf bifurcation as $h$ increases through $h \approx 0.07$. We use this information to indicate the stability of the branches in Fig. 12. This Hopf bifurcation appears to be subcritical. In Fig. 13 we show a simulation that starts with $h=0.05$, for which the moving pulse on the upper branch in Fig. 12 is stable. At $t=100, h$ is switched to 0.07 . The pulse starts to oscillate, but the oscillations grow in magnitude until the pulse is destroyed. Other families of travelling pulses are shown in Fig. 8 (red curves).

Supercritical Hopf bifurcations of moving pulses, leading to travelling breathers or "lurching" waves, have been observed in several other neural systems $[8,15]$. However, we could not find parameters for the system under study for which this type of bifurcation occurred.

\section{Other solutions}

In this section we discuss colliding fronts, "anti-pulses," and the patterns seen when inverted Mexican-hat connectivity is used. Returning to Fig. 9, we see that for these parameters and $h$ in some interval whose lower endpoint is $h=0.075$, if we were to start a wave consisting of a large active interval the "front" of the wave would move more slowly than the "back." The back would eventually catch up with the front. A simulation showing this can be seen in Fig. 14, and we see that the result is a moving pulse from the stable family shown in Fig. 8 (b).

If, however, we choose $h$ slightly less than 0.075 , the opposite will occur, i.e. the back moves more slowly than the front and the active region expands in width. On a periodic domain this occurs until the front meets the back, from behind, as seen in Fig. 15 (top). We call the final solution a moving "anti-pulse," since all but a small part of the domain is active.

The formation of a moving pulse by the catching up of a back to a front was seen in Ref. [7], but these authors did not mention the formation of anti-pulses, although they are 

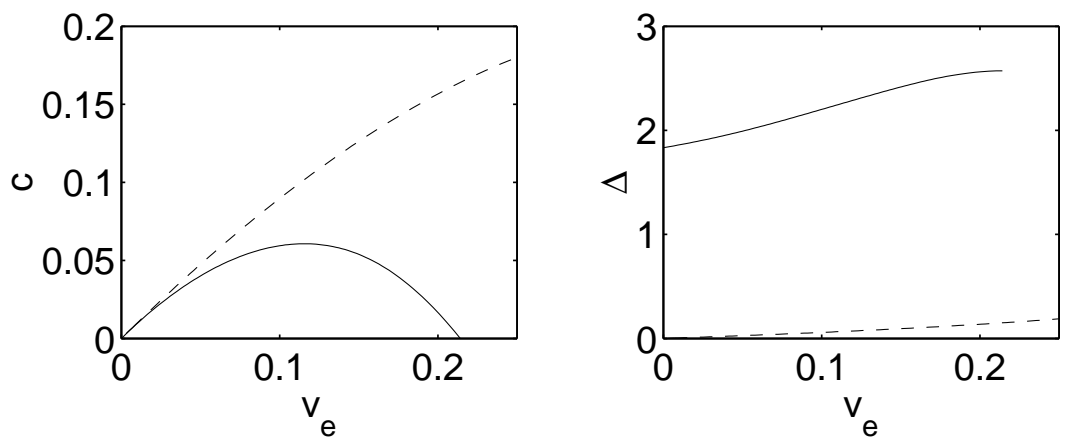

Figure 11: Left: pulse speeds as a function of $v_{e}$. Right: pulse widths as a function of $v_{e}$. Solid lines indicate stable solutions, dashed unstable. Note that the speeds are always less than $v_{e}$. Other parameters are $\alpha_{i}=\alpha_{e}=1, v_{i}=1, \sigma_{e}=1, \sigma_{i}=2, h=0.1, \Gamma=1$. 

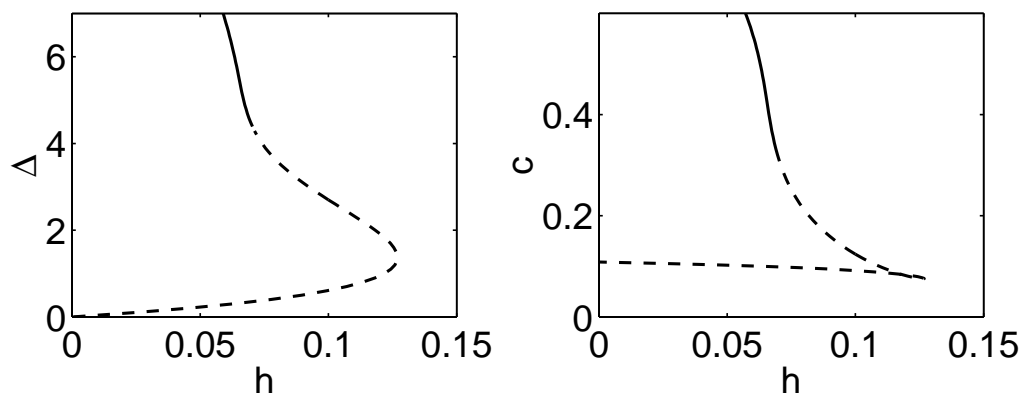

Figure 12: Width $(\Delta$, left panel) and speed ( $c$, right panel) of a moving pulse as function of $h$. A solid line indicates stability, while a dashed one indicates instability. There is a Hopf bifurcation on the top branch. Other parameters are $\Gamma=1, \sigma_{e}=1, \sigma_{i}=2, \alpha_{e}=1, \alpha_{i}=$ $0.3, v_{e}=v_{i}=1$. 


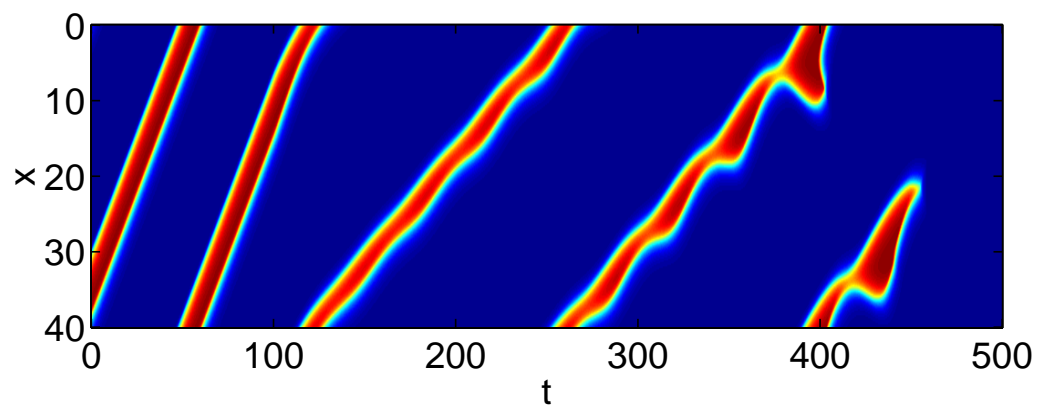

Figure 13: A subcritical Hopf bifurcation of a moving pulse. At $t=100, h$ is switched from 0.05 to 0.07 . $u_{e}$ is plotted, red high and blue low. Periodic boundary conditions are used. Other parameters are as in Fig. 12. 


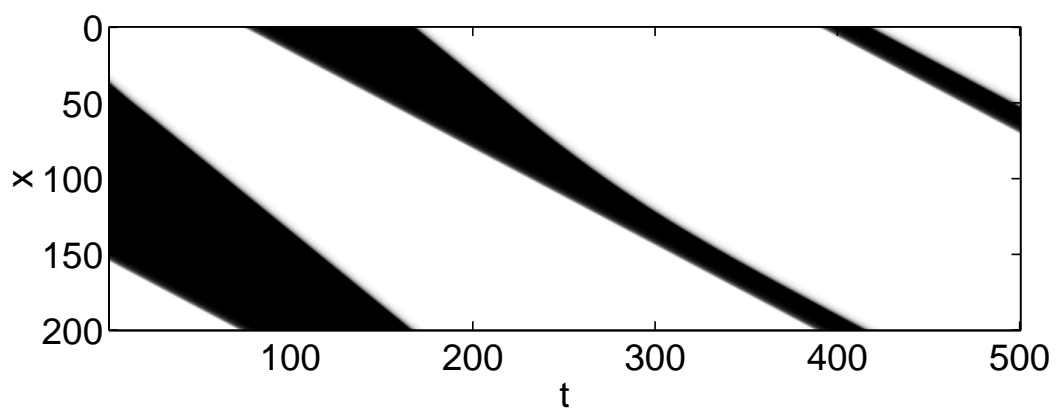

Figure 14: A wide moving pulse for which the back travels faster than the front. The eventual solution is a moving pulse, of the type analysed in Sec. 4.2. $u_{e}$ is plotted, with black being high and white low. Periodic boundary conditions are used. $h=0.115$ and other parameters are as in Fig. 9. 
most certainly expected even when $v_{e}=v_{i}$ and $\alpha_{e}=\alpha_{i}$. We now analyse anti-pulses.

\subsection{Anti-pulses}

For anti-pulses, $q(\xi)<h$ for $0<\xi<\Delta$ and $q(\xi)>h$ otherwise. Using $\int_{-\infty}^{\infty} w_{a}(x) d x=\Gamma_{a}$, we have

$$
\psi_{a}(\xi)= \begin{cases}\Gamma_{a}-\mathcal{F}_{a}\left(\frac{-\xi}{1+c / v_{a}}, \frac{\Delta-\xi}{1+c / v_{a}}\right) & \xi \leq 0 \\ \Gamma_{a}-\mathcal{F}_{a}\left(0, \frac{\xi}{1-c / v_{a}}\right)-\mathcal{F}_{a}\left(0, \frac{\Delta-\xi}{1+c / v_{a}}\right) & 0<\xi<\Delta, \\ \Gamma_{a}-\mathcal{F}_{a}\left(\frac{\xi-\Delta}{1-c / v_{a}}, \frac{\xi}{1-c / v_{a}}\right) & \xi \geq \Delta\end{cases}
$$

where $\mathcal{F}_{a}$ is given by (40). The conditions $q(0)=h=q(\Delta)$ are then easily determined using $q(\xi)=q_{e}(\xi)-q_{i}(\xi)$ and

$$
q_{a}(\xi)=\int_{0}^{\infty} \eta_{a}(s) \psi_{a}(\xi+c s) d s
$$

and the fact that these integrals have essentially been done in the determination of (41)-(42). They are

$$
\begin{aligned}
2 h= & -2\left[\Gamma \exp \left(-\alpha_{i} \Delta / c\right)-\exp \left(-\alpha_{e} \Delta / c\right)\right] \\
& -\left[\frac{\exp \left(-\alpha_{e} \Delta / c\right)-1}{1-c m_{e}^{-} / \alpha_{e}}\right]-\left[\frac{\exp \left(-\alpha_{e} \Delta / c\right)-\exp \left(-m_{e}^{+} \Delta\right)}{1-c m_{e}^{+} / \alpha_{e}}\right] \\
+ & \Gamma\left[\frac{\exp \left(-\alpha_{i} \Delta / c\right)-1}{1-c m_{i}^{-} / \alpha_{i}}\right]+\Gamma\left[\frac{\exp \left(-\alpha_{i} \Delta / c\right)-\exp \left(-m_{i}^{+} \Delta\right)}{1-c m_{i}^{+} / \alpha_{i}}\right]
\end{aligned}
$$

and

$$
2 h=2(1-\Gamma)-\left[\frac{1-\exp \left(m_{e}^{-} \Delta\right)}{1-c m_{e}^{-} / \alpha_{e}}\right]+\Gamma\left[\frac{1-\exp \left(m_{i}^{-} \Delta\right)}{1-c m_{i}^{-} / \alpha_{i}}\right]
$$

The width and speed of anti-pulses is determined by the simultaneous solution of (55)-(56). The Evans function for antipulses has the same form as that for pulses, since only the fact that $q(0)=q(\Delta)=h$ is used, the sign of $q(\xi)-h$ for $\xi \in(0, \Delta)$ being irrelevant.

In fact, the analysis of anti-pulses does not bring any new results, as the system under study is symmetric about the "balanced" parameter point $2 h=1-\Gamma$, at which there are stationary fronts (see Sec. 4.1). If we make the replacement $h \rightarrow 1-\Gamma-h$ in (55)-(56), we obtain (41)-(42), and vice versa. Thus, for a given value of $h$, say $h^{*}$, at which there exists a pulse, there exists a corresponding antipulse when $h=1-\Gamma-h^{*}$ (all other parameters being the same). It will have the same width (as determined by the zeros of $q(\xi)-h)$, speed and stability as the pulse.

\subsection{Inverted Mexican hat connectivity}

Although much work on pattern formation in neural field models has used one layer of neurons with Mexican-hat connectivity, for which inhibitory connections have a wider spatial extent than excitatory, there is evidence that the opposite is true, at least in some contexts [39]. We now briefly analyse (1)-(2) with coupling function (4), but with $\sigma_{e}>\sigma_{i}$, 

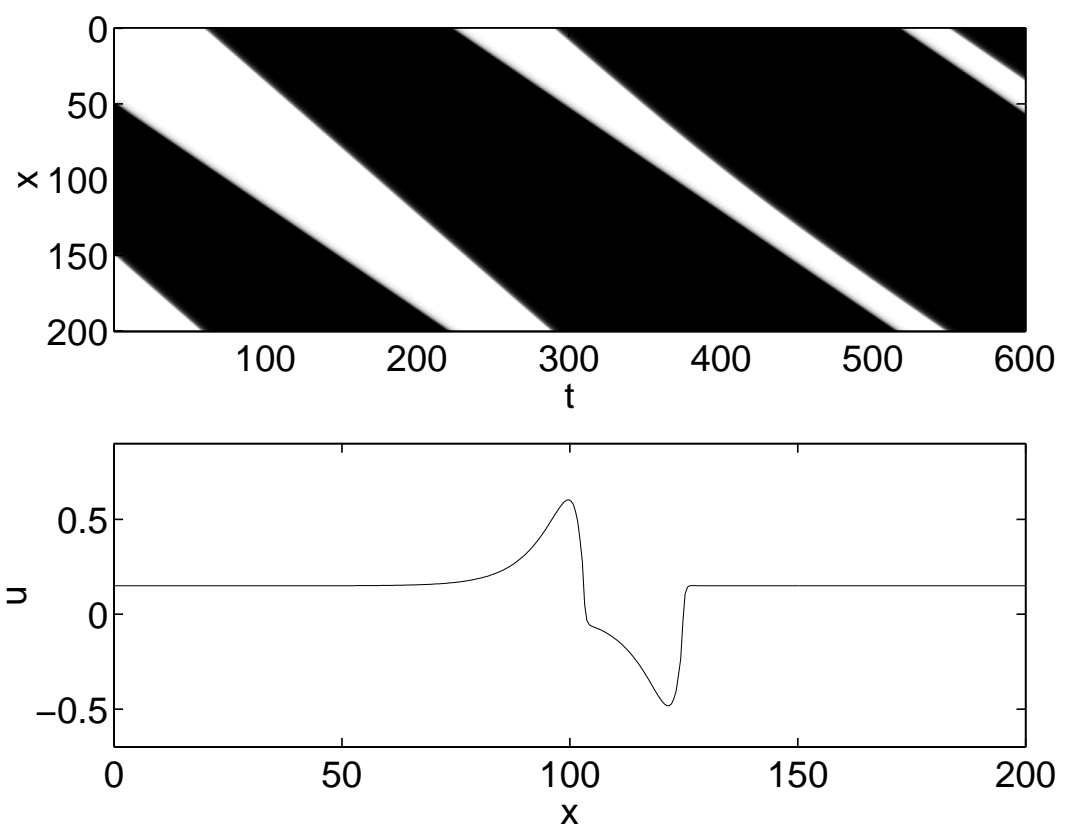

Figure 15: A wide moving pulse for which the back travels slower than the front, leading to the formation of an "anti-pulse". Top: $u_{e}$ is plotted, with black being high and white low. Bottom: $u=u_{e}-u_{i}$ once the anti-pulse has formed. $h=0.05$, so most of the domain is active. Other parameters are as in Fig. 9. 
i.e. with the excitatory connections having a greater spatial extent than the inhibitory, which we refer to as inverted Mexican-hat connectivity.

As before, we can find families of moving pulses. In Fig. 16 we show solutions of (41)(42) as $h$ is varied for inverted Mexican-hat connectivity. Note that not every point on the curves in Fig. 16 corresponds to a one-pulse solution, as some of the solutions may have $q(\xi)>h$ for more than one interval. In Fig. 17 we show a stable travelling one-pulse solution from the curve in Fig. 16 (top panel), and the complex transients that can occur when two such pulses interact (bottom panel, same parameters). For this connectivity, we can also obtain families of travelling fronts (not shown).

\section{Discussion}

We have studied stationary and travelling bump and front solutions of a two-layer neural field model with different conduction velocities and synaptic processing time-constants for the two populations. By varying these parameters we have found bifurcations of stationary bumps to both travelling and breathing bumps. These bifurcations can be found by explicitly constructing an Evans function for these solutions and, as shown in Sec. 3.4, they cannot occur if the synaptic time-constants and conduction velocities are the same for both layers.

Our work has produced results similar to those of several other groups. For example, Curtu and Ermentrout [9] recently studied an extension of the system first discussed by Hansel and Sompolinsky [19]. This model had one neural population, Mexican-hat type connectivity, an adaptation variable and no delays. The authors found travelling and standing waves, and stationary, spatially-periodic patterns. However, their results were derived by linearising about the spatially-uniform state, and are thus unable to say anything regarding spatially-localised patterns of the type studied here.

Golomb and Ermentrout [15] studied the effects of delays on propagating activity. They included a fixed delay and found that increasing this led to lurching waves. (We did not include a fixed delay, but see below.) However, they used a spiking neural network in which each neuron could only fire once, and only excitatory coupling. Because of this, they could not find stationary or arbitrarily slowly moving patterns, as we have. In later work [16, 17] these authors studied a network with both excitatory and inhibitory populations but did not include conduction delays for most of their analysis, and still allowed neurons to fire at most once, thus precluding the existence of stationary patterns. One interesting result that they found was the coexistence of both fast and slow propagating pulses, which we have not found. However, these authors found that once neurons were allowed to fire multiple times this bistability disappeared, with only the slow pulses persisting.

Blomquist et al. [1] studied a two-layer neuronal network without delays (an extension of that studied by Pinto and Ermentrout [33]), and found both subcritical and supercritical 

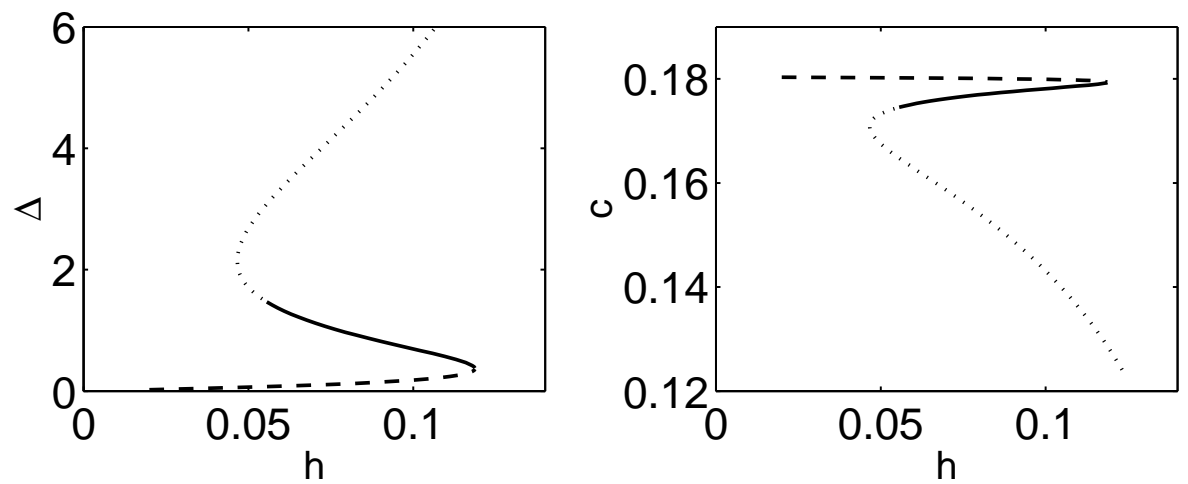

Figure 16: Width (left panel) and speed (right panel) of a travelling pulse as a function of $h$, with inverted Mexican hat connectivity. Solid lines represent stable one-bump solutions and dashed unstable, while the dotted lines indicate a solution of (41)-(42) which is not a one-bump solution. Parameters are $v_{e}=0.2, v_{i}=1, \alpha_{e}=1, \alpha_{i}=1, \Gamma=0.7, \sigma_{e}=2, \sigma_{i}=1$. 

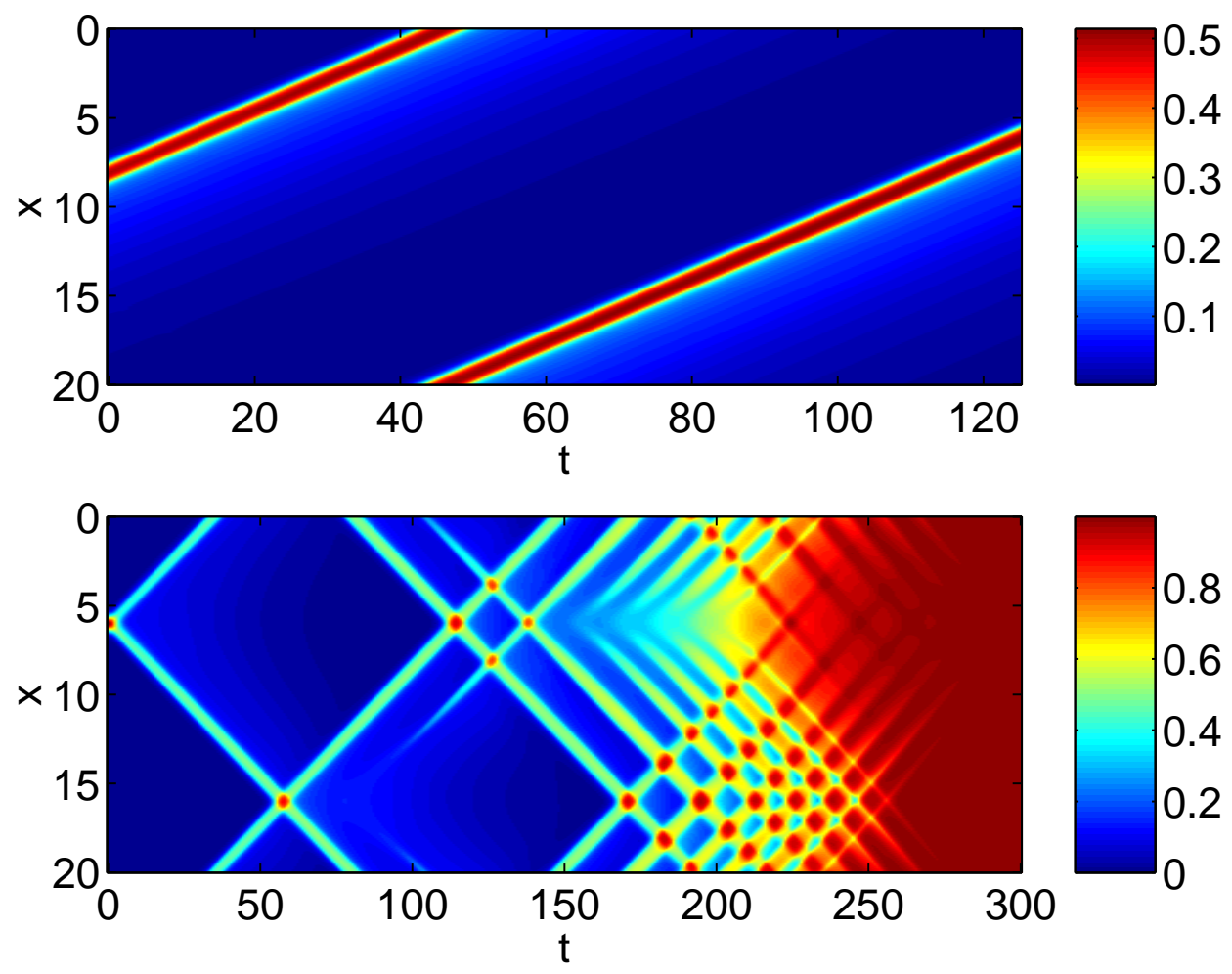

Figure 17: Top: a stable travelling pulse for inverted Mexican hat connectivity. Bottom: the interaction of two such travelling pulses leads to complex behavior. $u_{e}$ is plotted. Note the different time scales. Parameters are $v_{e}=0.2, v_{i}=1, \alpha_{e}=1, \alpha_{i}=1, \Gamma=0.7, \sigma_{e}=2, \sigma_{i}=$ $1, h=0.07$. 
Hopf bifurcations of stationary bumps. Coombes and Owen [8] studied a single neural layer with Mexican-hat connectivity and a variable representing spike frequency adaptation. They found both drifting and breathing bifurcations of stationary bumps, as we have, and also supercritical Hopf bifurcations of travelling bumps, which we have not found. We now discuss possible extensions of the work presented here.

One extension would be to break the homogeneity of the domain (reflected by the appearance of $x$ and $y$ in (2) in only the combination $x-y$ ) as Jirsa and Kelso have done [24], but for the system studied here. These authors modelled heterogeneity by putting a direct connection from one part of the domain to another and studied the effect of varying the length of this connection.

Roxin et al. [35] recently studied a generalisation of a neural field model first presented by Hansel and Sompolinsky [19] in which there is a fixed delay in the nonlinear term, as opposed to the space-dependent delays that we have. This delay is meant to mimic those due to synaptic and dendritic processing. These authors found a wide variety of spatiotemporal patterns. Including such a term in our equations would involve replacing $u\left(x-y, t-|y| / v_{a}\right)$ in (2) by $u\left(x-y, t-|y| / v_{a}-D\right)$, where $D$ is a fixed positive delay. As mentioned above, this type of delay was studied by Golomb and Ermentrout [15]. As an example of the effect of including such a term, it is straightforward to show that the equations governing the speed of a front (33)-(34) would be modified to

$$
\begin{array}{ll}
2 h=\frac{\exp \left(D c m_{e}^{-}\right)}{1-c m_{e}^{-} / \alpha_{e}}-\frac{\Gamma \exp \left(D c m_{i}^{-}\right)}{1-c m_{i}^{-} / \alpha_{i}} & c \geq 0 \\
2 h=2(1-\Gamma)+\frac{\Gamma \exp \left(D c m_{i}^{+}\right)}{1-c m_{i}^{+} / \alpha_{i}}-\frac{\exp \left(D c m_{e}^{+}\right)}{1-c m_{e}^{+} / \alpha_{e}} & c<0
\end{array}
$$

and it seems likely that all of the calculations performed here could also be done with such a term included. Hutt [21] discussed a similar idea, but wrote the nonlinear term in (2) as a linear combination of a term whose delay depends on distance and one whose delay is fixed.

Further extensions could include verifying some of the predictions here with a network of spiking neurons. This would be computationally intensive due to the inclusion of delays, but similar work has been performed [15]. An important extension that would make more valid the results here and elsewhere $[7,8]$, would be to derive similar results for a continuous firing rate function, $f$ [9]. Also important is the consideration of a two-dimensional domain since the cortex is best thought of as a two-dimensional sheet of interconnected neurons. There have been recent results on patterns (multi-bump solutions, breathers and spiral waves) in two-dimensional neural fields [30, 13, 28], but none of these models have included even one conduction velocity or delay. While it seems that including a finite conduction velocity does not destabilise travelling bumps or fronts in one spatial dimension [5, 15], it is not clear whether the same holds in two dimensions. 
A more general model, more clearly differentiating the two neural populations, would be

$$
\begin{aligned}
u_{a}=\eta_{a} * \psi_{a}, \quad a \in\{e, i\} & \\
\psi_{e}(x, t)= & \int_{-\infty}^{\infty} \mathrm{d} y w_{e e}(y) f_{e} \circ u_{e}\left(x-y, t-|y| / v_{e}\right) \\
& -\int_{-\infty}^{\infty} \mathrm{d} y w_{i e}(y) f_{i} \circ u_{i}\left(x-y, t-|y| / v_{i}\right) \\
\psi_{i}(x, t)= & \int_{-\infty}^{\infty} \mathrm{d} y w_{e i}(y) f_{e} \circ u_{e}\left(x-y, t-|y| / v_{e}\right) \\
& -\int_{-\infty}^{\infty} \mathrm{d} y w_{i i}(y) f_{i} \circ u_{i}\left(x-y, t-|y| / v_{i}\right)
\end{aligned}
$$

Here we not only have different conduction velocities $v_{e}$ and $v_{i}$ and different synaptic filters $\eta_{e}$ and $\eta_{i}$, but different firing rate functions $f_{e}$ and $f_{i}$ and four coupling functions, $w_{e e}, w_{e i}, w_{i e}$ and $w_{i i}$ instead of the two in (1)-(2). Choosing $f_{a}(u)=\Theta\left(u-h_{a}\right)$, i.e. using the Heaviside function as the firing rate function, with two different thresholds, we should be able to analyse (59)-(61) in much the same way as we have analysed (1)-(2) in this paper. Some of the analysis in [8], in which the threshold is a dynamic variable, should be applicable to the analysis of (59)-(61) when $h_{e} \neq h_{i}$. The model (1)-(2) can be considered as intermediate between (59)-(61) and previous models in which there is only one population of neurons, one synaptic filter, and one conduction velocity $[6,7]$.

Guo et al. [18] have recently studied a pair of coupled delay-differential equations that are similar in structure to (59)-(61), as have Shayer and Campbell [36], although those systems have no spatial structure. Note that after setting $v_{e}=v_{i}=\infty$ in (60)-(61) and choosing $\eta_{e}(t)=\Theta(t) e^{-t}$ and $\eta_{i}(t)=\Theta(t) e^{-t / \tau} / \tau$ we recover the model originally presented by Pinto and Ermentrout [33] and later analysed by Blomquist et al. [1].

Acknowledgements: We thank the referees for their comments. CRL was supported by the Marsden Fund, administered by the Royal Society of New Zealand. This work was initiated during a visit by CRL to the University of Nottingham, supported by a grant to SC from the London Mathematical Society. SC would also like to acknowledge ongoing support from the EPSRC through the award of an Advanced Research Fellowship, Grant No. GR/R76219.

\section{References}

[1] P Blomquist, J Wyller, and G T Einevoll. Localized activity patterns in two-population neuronal networks. Physica D, 206:180-212, 2005.

[2] P C Bressloff. Traveling waves and pulses in a one-dimensional network of excitable integrate-and-fire neurons. Journal of Mathematical Biology, 40:169-198, 2000.

[3] P C Bressloff and S E Folias. Front-bifurcations in an excitatory neural network. SIAM Journal on Applied Mathematics, 65:131-151, 2004. 
[4] P C Bressloff, S E Folias, A Prat, and Y X Li. Oscillatory waves in inhomogeneous neural media. Physical Review Letters, 91:178101, 2003.

[5] S Coombes. Waves and bumps in neural field theories. Biological Cybernetics, 93:91-108, 2005.

[6] S Coombes, G J Lord, and M R Owen. Waves and bumps in neuronal networks with axo-dendritic synaptic interactions. Physica D, 178:219-241, 2003.

[7] S Coombes and M R Owen. Evans functions for integral neural field equations with Heaviside firing rate function. SIAM Journal on Applied Dynamical Systems, 34:574-600, 2004.

[8] S Coombes and M R Owen. Bumps, breathers, and waves in a neural network with spike frequency adaptation. Physical Review Letters, 94:148102, 2005.

[9] R Curtu and B Ermentrout. Pattern formation in a network of excitatory and inhibitory cells with adaptation. SIAM Journal on Applied Dynamical Systems, 3:191-231, 2004.

[10] G B Ermentrout and D Kleinfeld. Traveling electrical waves in cortex: insights from phase dynamics and speculation on a computational role. Neuron, 29:33-44, 2001.

[11] G B Ermentrout and J B McLeod. Existence and uniqueness of travelling waves for a neural network. Proceedings of the Royal Society of Edinburgh, 123A:461-478, 1993.

[12] J Evans. Nerve axon equations: IV The stable and unstable impulse. Indiana University Mathematics Journal, 24:1169-1190, 1975.

[13] S E Folias and P C Bressloff. Breathing pulses in an excitatory neural network. SIAM Journal on Applied Dynamical Systems, 3:378-407, 2004.

[14] P S Goldman-Rakic. Cellular basis of working memory. Neuron, 14:477-485, 1995.

[15] D Golomb and G B Ermentrout. Effects of delay on the type and velocity of travelling pulses in neuronal networks with spatially decaying connectivity. Network: Computation in Neural Systems, 11:221-246, 2000.

[16] D Golomb and G B Ermentrout. Bistability in pulse propagation in networks of excitatory and inhibitory populations. Physical Review Letters, 86:4179-4182, 2001.

[17] D Golomb and G B Ermentrout. Slow excitation supports propagation of slow pulses in networks of excitatory and inhibitory populations. Physical Review E, 65:061911, 2002.

[18] S Guo, L Huang, and J Wu. Regular dynamics in a delayed network of two neurons with all-or-none activation functions. Physica D, 206:32-48, 2005. 
[19] D Hansel and H Sompolinsky. Methods in neuronal modeling: from ions to networks, pages 499-567. MIT Press, Cambridge, MA, 1998.

[20] X Huang, W C Troy, Q Yang, H Ma, C R Laing, S J Schiff, and J Wu. Spiral waves in disinhibited mammalian neocortex. The Journal of Neuroscience, 24:9897-9902, 2004.

[21] A Hutt. Effects of nonlocal feedback on traveling fronts in neural fields subject to transmission delay. Physical Review E, 70:052902, 2004.

[22] A Hutt, M Bestehorn, and T Wennekers. Pattern formation in intracortical neuronal fields. Network: Computation in Neural Systems, 14:351-368, 2003.

[23] V K Jirsa and H Haken. Field theory of electromagnetic brain activity. Physical Review Letters, 77:960-963, 1996.

[24] V K Jirsa and J A S Kelso. Spatiotemporal pattern formation in neural systems with heterogeneous connection topologies. Physical Review E, 62:8462-8465, 2000.

[25] T Kapitula. Dissipative Solitons, chapter Stability analysis of pulses via the Evans function: dissipative systems, pages 407-428. Springer-Verlag, 2005.

[26] T Kapitula, N Kutz, and B Sandstede. The Evans function for nonlocal equations. Indiana University Mathematics Journal, 53:1095-1126, 2004.

[27] C Koch. Biophysics of computation: information processing in single neurons. Oxford University Press, 1999.

[28] C R Laing. Spiral waves in nonlocal equations. SIAM Journal on Applied Dynamical Systems, 4:588-606, 2005.

[29] C R Laing and A Longtin. Noise induced stabilization of bumps in systems with long range spatial coupling. Physica D, 160:149-172, 2001.

[30] C R Laing and W C Troy. PDE methods for nonlocal models. SIAM Journal on Applied Dynamical Systems, 2:487-516, 2003.

[31] B A McGuire, C D Gilbert, P K Rivlin, and T N Wiesel. Targets of horizontal connections in macaque primary visual cortex. Journal of Comparative Neurology, 305:370-392, 1991.

[32] D J Pinto and G B Ermentrout. Spatially structured activity in synaptically coupled neuronal networks: I. Travelling fronts and pulses. SIAM Journal on Applied Mathematics, 62:206-225, 2001.

[33] D J Pinto and G B Ermentrout. Spatially structured activity in synaptically coupled neuronal networks: II. Lateral inhibition and standing pulses. SIAM Journal on Applied Mathematics, 62:226-243, 2001. 
[34] D J Pinto, R K Jackson, and C E Wayne. Existence and stability of traveling pulses in a continuous neuronal network. SIAM Journal on Applied Dynamical Systems, 4:954-984, 2005.

[35] A Roxin, N Brunel, and D Hansel. Role of delays in shaping spatiotemporal dynamics of neuronal activity in large networks. Physical Review Letters, 94:238103, 2005.

[36] L P Shayer and S A Campbell. Stability, bifurcation, and multistability in a system of two coupled neurons with multiple time delays. SIAM Journal on Applied Mathematics, 61:673-700, 2000.

[37] H A Swadlow. Physiological properties of individual cerebral axons studied in vivo for as long as one year. Journal of Neurophysiology, 54:1346-1362, 1985.

[38] H A Swadlow. Efferent neurons and suspected interneurons in s-1 forelimb representation of the awake rabbit: receptive fields and axonal properties. Journal of Neurophysiology, 63:1477-1498, 1990.

[39] N V Swindale. The development of topography in the visual cortex: a review of models. Network: Computation in Neural Systems, 7:161-247, 1996.

[40] N V Swindale. Neural synchrony, axonal path lengths and general anesthesia: A hypothesis. The Neuroscientist, 9:440-445, 2003.

[41] S Wiggins. Introduction to applied nonlinear dynamical systems and chaos. Springer-Verlag, New York, 1990. 